\title{
Article \\ Familial Occurrence of Adult Granulosa Cell Tumors: Analysis of Whole-Genome Germline Variants
}

\author{
Joline F. Roze ${ }^{1, *}$, Joachim Kutzera ${ }^{2}$, Wouter Koole ${ }^{2}$, Margreet G. E. M. Ausems ${ }^{2}$, Kristi Engelstad ${ }^{3}$, \\ Jurgen M. J. Piek ${ }^{4}{ }^{(D)}$, Cor D. de Kroon ${ }^{5}{ }^{D}$, René H. M. Verheijen ${ }^{1}$, Gijs van Haaften ${ }^{2}$, Ronald P. Zweemer ${ }^{1, *}$ \\ and Glen R. Monroe ${ }^{1}$
}

check for updates

Citation: Roze, J.F.; Kutzera, J.;

Koole, W.; Ausems, M.G.E.M.;

Engelstad, K.; Piek, J.M.J.; de Kroon, C.D.; Verheijen, R.H.M.; van Haaften,

G.; Zweemer, R.P.; et al. Familial

Occurrence of Adult Granulosa Cell

Tumors: Analysis of Whole-Genome Germline Variants. Cancers 2021, 13, 2430. https://doi.org/10.3390/ cancers13102430

Academic Editors: Mohamed

Mokhtar Desouki and

Oluwole Fadare

Received: 14 April 2021

Accepted: 14 May 2021

Published: 18 May 2021

Publisher's Note: MDPI stays neutral with regard to jurisdictional claims in published maps and institutional affiliations.

Copyright: (c) 2021 by the authors. Licensee MDPI, Basel, Switzerland. This article is an open access article distributed under the terms and conditions of the Creative Commons Attribution (CC BY) license (https:// creativecommons.org/licenses/by/ $4.0 /)$.
1 Department of Gynaecological Oncology, UMC Utrecht Cancer Center, University Medical Center Utrecht, Utrecht University, 3584 CX Utrecht, The Netherlands; rene.h.m.verheijen@gmail.com (R.H.M.V.); Gmonroe1@its.jnj.com (G.R.M.)

2 Department of Genetics, University Medical Center Utrecht, Utrecht University, 3584 CX Utrecht, The Netherlands; J.Kutzera@umcutrecht.nl (J.K.); W.Koole@umcutrecht.nl (W.K.); M.G.E.M.Ausems@umcutrecht.nl (M.G.E.M.A.); G.vanHaaften@umcutrecht.nl (G.v.H.)

3 FM Ambulance, Fargo, ND 58078, USA; kristi.engelstad@fmambulance.com

4 Department of Obstetrics and Gynaecology, Catharina Hospital, 5623 EJ Eindhoven, The Netherlands; jurgen.piek@catharinaziekenhuis.nl

5 Department of Obstetrics and Gynaecology, Leiden University Medical Center, 2333 ZA Leiden, The Netherlands; C.D.de_Kroon@lumc.nl

* Correspondence: J.F.Roze-2@umcutrecht.nl (J.F.R.); R.Zweemer@umcutrecht.nl (R.P.Z.); Tel.: +88-755-5555 (J.F.R. \& R.P.Z.)

Simple Summary: Although granulosa cell tumors can occur in rare syndromes and one familial case of a granulosa cell tumor has been described, a genetic predisposition for granulosa cell tumors has not been identified. Through our collaborations with patients, we identified four families in which two women of each family were diagnosed with an adult granulosa cell tumor. Although predicted deleterious variants in PIK3C2G, BMP5, and LRP2 were found, we could not identify an overlapping genetic variant or affected locus that may explain a genetic predisposition for granulosa cell tumors. The age of onset in the familial patients was significantly lower (median 38 years, range from 17 to 60) than in sporadic patients (median between 50 and 55 years). Furthermore, breast cancer, polycystic ovary syndrome, and subfertility were seen in these families.

Abstract: Adult granulosa cell tumor (AGCT) is a rare ovarian cancer subtype, with a peak incidence around 50-55 years. Although AGCT can occur in specific syndromes, a genetic predisposition for AGCT has not been identified. The aim of this study is to identify a genetic variant in families with AGCT patients, potentially contributing to tumor evolution. We identified four families, each including two women diagnosed with AGCT. Whole-genome sequencing was performed to identify overlapping germline variants or affected genes. Familial relationship was evaluated using genealogy and genomic analyses. Patient characteristics, medical (family) history, and pedigrees were collected. Findings were compared to a reference group of 33 unrelated AGCT patients. Mean age at diagnosis was 38 years (range from 17 to 60 ) versus 51 years in the reference group, and seven of eight patients were premenopausal. In two families, three first degree relatives were diagnosed with breast cancer. Furthermore, polycystic ovary syndrome (PCOS) and subfertility was reported in three families. Predicted deleterious variants in PIK3C2G, BMP5, and LRP2 were identified. In conclusion, AGCTs occur in families and could potentially be hereditary. In these families, the age of AGCT diagnosis is lower and cases of breast cancer, PCOS, and subfertility are present. We could not identify an overlapping genetic variant or affected locus that may explain a genetic predisposition for AGCT.

Keywords: granulosa cell tumor; sex cord-stromal tumors; ovarian cancer; whole-genome sequencing; FOXL2 


\section{Introduction}

Ovarian cancer is the fifth leading cause of cancer-related death among women and arises from epithelial, sex-cord stromal or germ cells [1]. Granulosa cell tumors belong to the sex-cord stromal tumors and represent $5 \%$ of ovarian cancers, with an estimated incidence of $0.6-1.0$ in 100,000 women worldwide per year [2]. The tumor is derived from the hormonally active granulosa cells that produce estradiol. Patients may develop symptoms, such as vaginal bleeding, caused by prolonged estrogen exposure or abdominal pain. Occasionally, a granulosa cell tumor is diagnosed in patients presenting with subfertility, potentially as a result of unregulated inhibin secretion by the tumor [3,4]. Although granulosa cell tumors can occur at any age, they mostly present perimenopausal or early in postmenopause, with a median age of diagnosis between 50 and 54 years [2]. Granulosa cell tumors are subdivided into an adult type ( $95 \%$ ) and juvenile type $(5 \%)$ by their histological and molecular characteristics [5], the latter mainly occurring at a younger age.

Germline mutations are involved in the evolution of some ovarian cancers. Approximately $10-15 \%$ of epithelial ovarian cancer is caused by a germline mutation in the BRCA1 or $B R C A 2$ gene [6]. In addition, specific hereditary syndromes result in an increased risk for sex cord-stromal tumors. Peutz-Jeghers syndrome is a rare autosomal dominant disease caused by germ line mutations in STK11/LKB1. Mutations in this gene are associated with gastrointestinal polyps, pigmentation of lips, and ovarian granulosa, and Sertoli cell tumors [7]. In addition, a germline mutation in DICER1 can cause a hereditary syndrome that is associated with Sertoli-Leydig cell tumors [8]. Furthermore, Olliers disease and Maffucci syndrome are rare disorders caused by early post-zygotic mutations in IDH1 and IDH2 genes and are associated with juvenile granulosa cell tumors [9].

Adult granulosa cell tumors (AGCTs) harbor a specific somatic FOXL2 c.402C > G mutation in approximately $95 \%$ of cases [10]. The $\mathrm{C} 134 \mathrm{~W}$ protein change caused by this mutation leads to reduced apoptosis, although the mechanism of granulosa cell tumorigenesis has not yet been entirely unraveled [11]. FOXL2 is preferentially expressed in the ovary, the eyelids, and the pituitary gland. Inactivating germline mutations in this gene do not result in AGCTs but in the autosomal dominant blepharophimosis-ptosis-epicanthus inversus syndrome (BPES) type I [12]. This disease affects the eyelids and is associated with granulosa cell dysfunction and premature ovarian failure.

Despite the fact that rare autosomal disorders are associated with the development of granulosa cell tumors, there is no known genetic predisposition that is specific for AGCT. To date, there has been only one reported case of a family in which both mother and daughter were diagnosed with a granulosa cell tumor [13]. This was seen as a coincidental finding and DNA analysis was not performed. We identified four different families in which two relatives were diagnosed with a granulosa cell tumor.

In this study, we performed whole-genome sequencing on familial AGCT patients to investigate overlapping germline mutations or shared affected genes as a potential cause for AGCT development. Findings were compared to a reference group of 33 unrelated AGCT patients [14]. Identification of an overlapping germline variant or affected genetic locus in these families could help to unravel the pathological mechanism of tumor evolution in AGCT patients.

\section{Materials and Methods}

We identified four different families that each had two women previously diagnosed with an AGCT. Patients were identified through contact with the national and international granulosa cell tumor patient organization and gynecological oncologists involved in our national research on granulosa cell tumors [15]. Ethical approval was obtained from the Institutional Review Board of the University Medical Center Utrecht (UMCU METC 17-868). All participants provided written informed consent. Peripheral blood samples $(n=6)$ or saliva swabs $(n=2)$ were collected for germline DNA analysis. DNA isolation and sequencing was performed according to previously described methods [14]. Clinical data were provided by the treating gynecologists $(n=6)$ or by the patients themselves 
$(n=2)$, including the age at diagnosis, disease stage, medical history, and family history. We retrieved information on the family relations and on the medical history of non-affected family members, in order to build pedigrees. A genealogist traced back family lineages to investigate whether a relationship between the four families existed. In addition, we used TRIBES to assess genetic relatedness within and between families. TRIBES is a pipeline for relatedness detection in genomic data, using the 1000 Genomes European cohort, which can accurately assess genetic relatedness up to 7th degree relatives [16]. Additionally, we used the whole-genome sequencing results of germline DNA from 33 unrelated AGCT patients as a reference group [14]. We tested for potential relatedness within and between the reference group and the families. Furthermore, we investigated whether clinical characteristics (i.e., age of onset and disease stage) differed between the related AGCT patients and the unrelated reference group and checked whether overlapping germline variants or affected genes present in the germline DNA of the families, were also present in the germline DNA of the reference group.

\subsection{Whole-Genome Sequencing and Variant Calling}

Whole-genome sequencing was performed with $30 \times$ coverage on Illumina HiSeq $X$ or NovaSeq 6000 instrument (Illumina, San Diego, CA, USA) by the Hartwig Medical Foundation (HMF, Amsterdam, NL, USA). Genome analysis was performed using the UMC IAP pipeline [17]. Sequence reads were mapped with Burrows-Wheeler Alignment v0.75a against human reference genome GRCh37. Single nucleotide variants and small insertions and deletions were called with GATK (v3.4.46). The functional effect of these variants was predicted with SnpEff (v.4.1). Structural variants were called using DELLY (0.7.2) [18] and Manta(v0.29.5).

\subsection{Genome Analysis}

First, all coding variants shared within families were analyzed. Variants with a high population frequency (>1:1000 according to population databases [19-22]) were filtered out, as AGCT constitutes a rare malignancy. We further removed frequent variants and sequencing artefacts using an in-house cancer reference database from the Hartwig Medical Foundation (HMF pool of normal variants V2.0, a resource with 78655034 unique variant calls from 1762 individuals) [23]. The exonic ( \pm 10 base pairs into the intronic regions) variant analysis was performed using Alissa (Agilent Technologies Alissa Interpret v5.1.7). Furthermore, variants present in the COSMIC Cancer Gene Census (release v89) were annotated and reviewed (Table S1). We assessed all variants present in cancer genes in the Human Gene Mutation Database (HGMD ${ }^{\circledR}$ Professional 2020.3) to identify potential inherited disease-causing mutations. Finally, variants genome-wide were ranked on their potential pathogenicity by using the Combined Annotation Dependent Depletion (CADD PHRED) score [24]. Variants with a deleteriousness score $\geq 5$ were annotated (Table S1), and intra-family variants with a score $\geq 20$ were assessed.

Second, we investigated overlapping genome-wide variants and affected genes between families. To assess shared variants, we selected variants present in both individuals in at least one family, and all variants shared by $\geq 2$ families were evaluated by predicted pathogenicity of the variant and gene function. Additionally, we searched for recurrently affected genes across different families and evaluated them on mutation effect and gene function.

Third, we investigated larger structural variations across samples. Structural variants present in the GNOMAD catalogue [25] and the HMF SV pool of normals were removed prior to analysis. We used in-house scripts based on the R-package StructuralVariantAnnotation to analyze the samples [26]. Shared breakpoints were determined using the function "findBreakpointOverlaps" from StructuralVariantAnnotation with default parameters, and variants with overlapping breakpoints between samples were grouped together. Structural variants present in less than one family were removed. We annotated the remaining struc- 
tural variants, the genes present within each structural variant region and investigated those genes for known involvement in granulosa cells or cancer development (Table S1).

\section{Results}

\subsection{Description of Families}

We identified four families with two women diagnosed with AGCT, three originating from the Netherlands and one American family. Pedigrees are shown in Figure 1. Family lineages were traced back for at least seven generations. All families (for 7-9 generations) came from different geographical areas and did not have any overlapping family names. Furthermore, their genomic relatedness correlated directly with their actual relatedness based on the pedigrees and there was no close genetic relationship between families (all predicted $\geq 9$ th degree relatedness according to TRIBES, see Table S2). None of the families were linked, indicating families did not share a recent common ancestor.
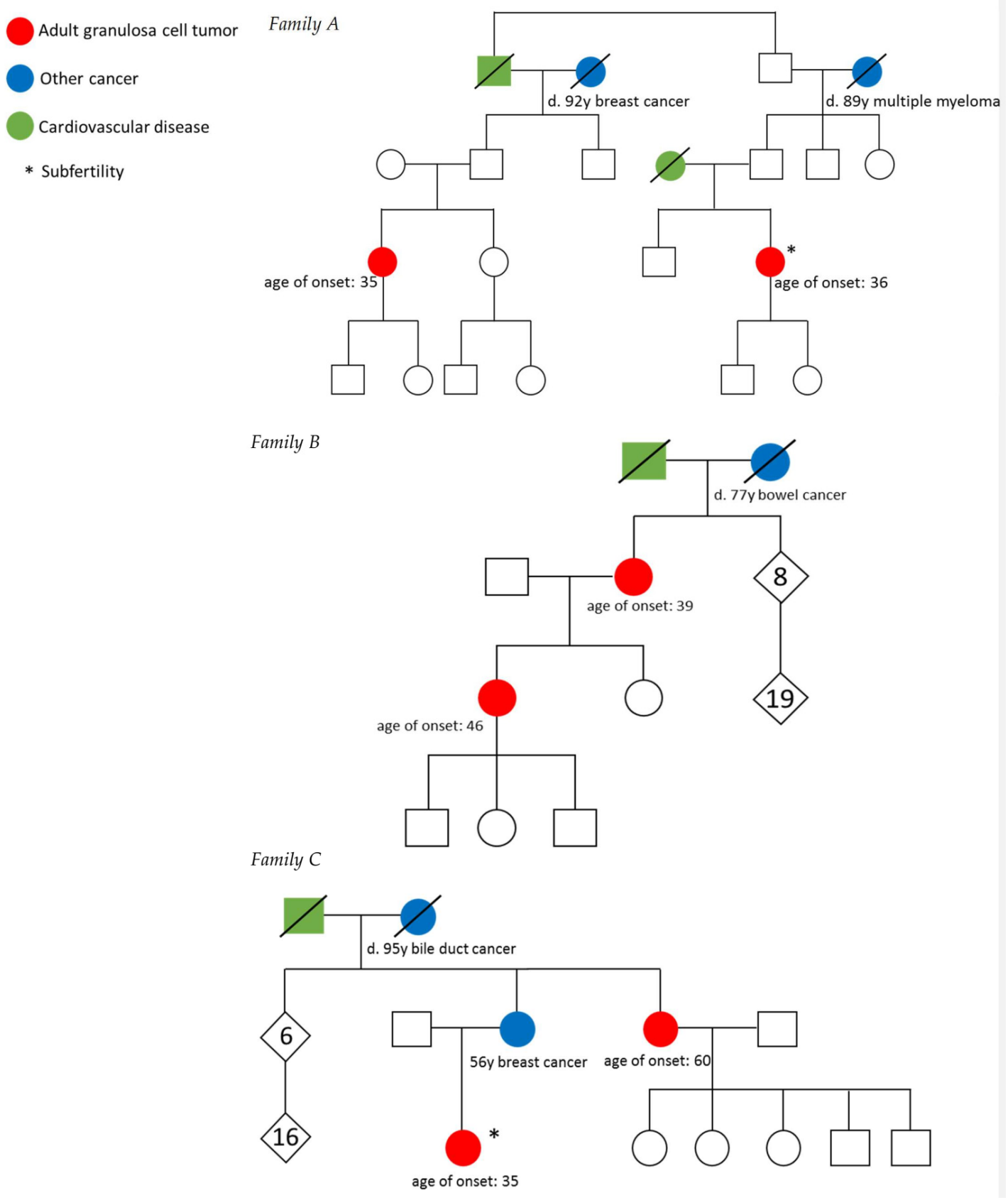

Figure 1. Cont. 


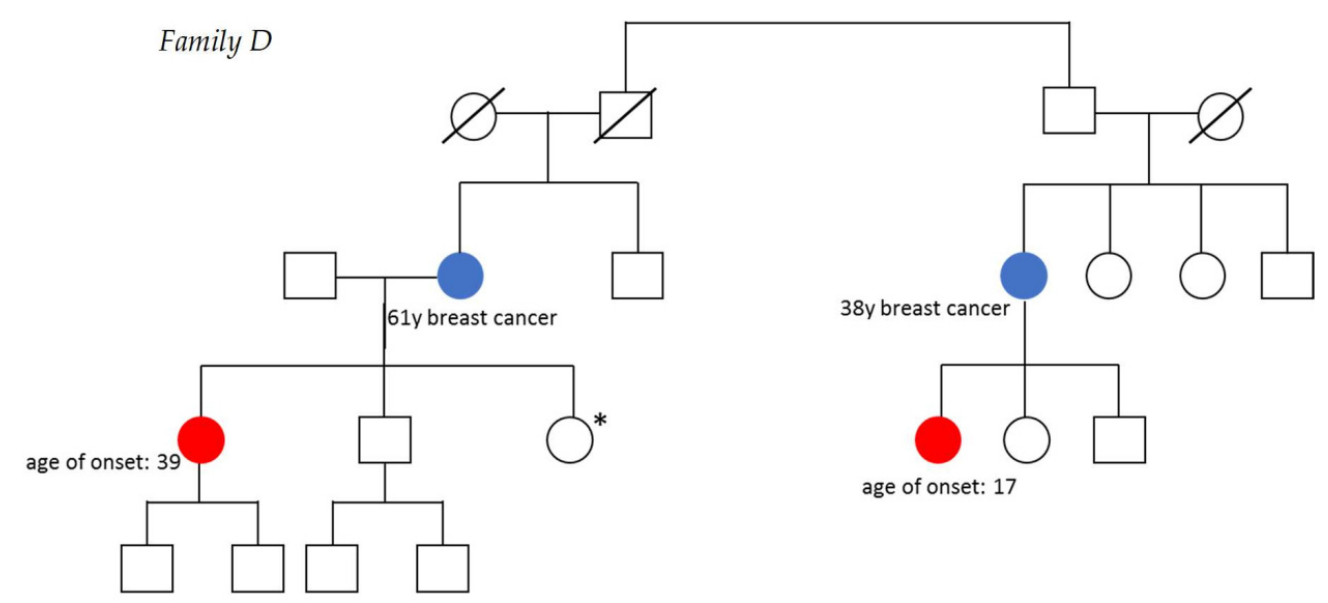

Figure 1. Family pedigrees. Colors represent diagnosis of AGCT, other cancer or cardiovascular disease. Deceased persons are indicated with a slash (/). Family A. Patients were related via their grandfathers. Family B. The AGCT patients represented mother and daughter. Family $\mathrm{C}$. The AGCT patients represented niece and aunt. Their mother, respectively sister was diagnosed with breast cancer. Patient C2 was involuntarily childless because of subfertility and more cases of subfertility were reported in this family. Family D. The AGCT patients were related through their grandfathers, who were brothers. Both mothers of the patients were diagnosed with breast cancer. One of the patient's sisters was also involuntarily childless due to subfertility.

Family A included two women with an AGCT, who were fifth-degree relatives as their grandfathers were brothers. The patients were diagnosed with stage IIB and IA AGCT at 35 and 36 years of age, respectively. The medical history revealed congenital clubfoot in the first patient and subfertility associated with polycystic ovary syndrome in the other patient (Table 1). Family B contained two first-degree relatives, mother and daughter, both diagnosed with AGCT at the age of 39 and 46, respectively. The mother underwent surgery and radiotherapy for stage IC disease and the daughter was treated with surgery only for stage IC AGCT. The five sisters and three brothers of the affected mother, as well as their offspring (in total 19 children), were all unaffected by any cancer type. Family C included two second-degree relatives (aunt and niece) diagnosed with stage IA AGCT at 35 and 60 years of age, respectively. The first patient was diagnosed after unsuccessful subfertility treatment, as she suffered from polycystic ovary syndrome. More cases of subfertility were reported in this family. The respective mother and sister of the affected patients was diagnosed with breast cancer when she was 56 years old. Family D consisted of two fifth-degree relatives, related via their grandfathers, diagnosed with stage IC AGCT at the age of 17 and 39 years. The mothers of the affected patients had a history of breast cancer and were diagnosed at 38 and 61 years of age. Furthermore, one of the patient's sisters was unable to conceive. All patients were successfully treated with surgery $(n=5)$, with surgery and radiotherapy $(n=1)$, or with surgery and chemotherapy $(n=2)$. None of the patients developed a recurrence during a median follow up time of 6 years (range from 4 to 38 years). In summary, polycystic ovary syndrome (PCOS) and subfertility were reported in three families and three first degree relatives had a history of breast cancer.

\subsection{Exome Analysis}

Within the families, 26 to 202 shared coding germline variants (exonic $+/-10$ base pairs) were detected (Figure 2). Exonic variants passing quality filters that resulted in a frameshift, stop/start loss, or a nonsynonymous variant predicted to be pathogenic from $3 / 5$ pathogenicity algorithms were retained $(n=93)$ (Table S1). Candidate genes affected by these variants in individual families are listed in Table 2. Furthermore, we detected 143 variants in 66 cancer-associated genes, including BMP5, with the highest predicted deleteriousness score of 22.7, MET and USP44 (Table S1). None of the detected variants in cancer genes were officially classified as inherited germline disease-causing mutations (HGMD ${ }^{\circledR}$ Professional 2020.3). 
Table 1. Patient characteristics.

\begin{tabular}{|c|c|c|c|c|c|}
\hline Patient & Age at Diagnosis & Tumor Stage & Treatment $* *$ & Medical History & Family History \\
\hline A1 & 35 & IIB * & Surgery & Clubfoot & \\
\hline $\mathrm{A} 2$ & 36 & IA & Surgery & $\begin{array}{c}\text { Polycystic ovary } \\
\text { syndrome, } 2 x \text { vaginal } \\
\text { delivery after in vitro } \\
\text { fertilization }\end{array}$ & \\
\hline B1 & 46 & IC & Surgery & & \\
\hline B2 & 39 & Unknown & Surgery and radiotherapy & & \\
\hline $\mathrm{C} 1$ & 35 & IA & Surgery & $\begin{array}{c}\text { Polycystic ovary } \\
\text { syndrome, subfertility }\end{array}$ & $\begin{array}{c}\text { Breast cancer, } \\
\text { PCOS, subfertility }\end{array}$ \\
\hline $\mathrm{C} 2$ & 60 & IA & Surgery & & \\
\hline D1 & 17 & IC & Surgery and chemotherapy & & $\begin{array}{l}\text { Breast cancer, } \\
\text { subfertility }\end{array}$ \\
\hline D2 & 39 & IC & Surgery and chemotherapy & & \\
\hline
\end{tabular}

${ }^{*}$ location dorsal from the uterus. ${ }^{* *}$ no recurrences occurred, and all patients were currently alive with no evidence of disease.

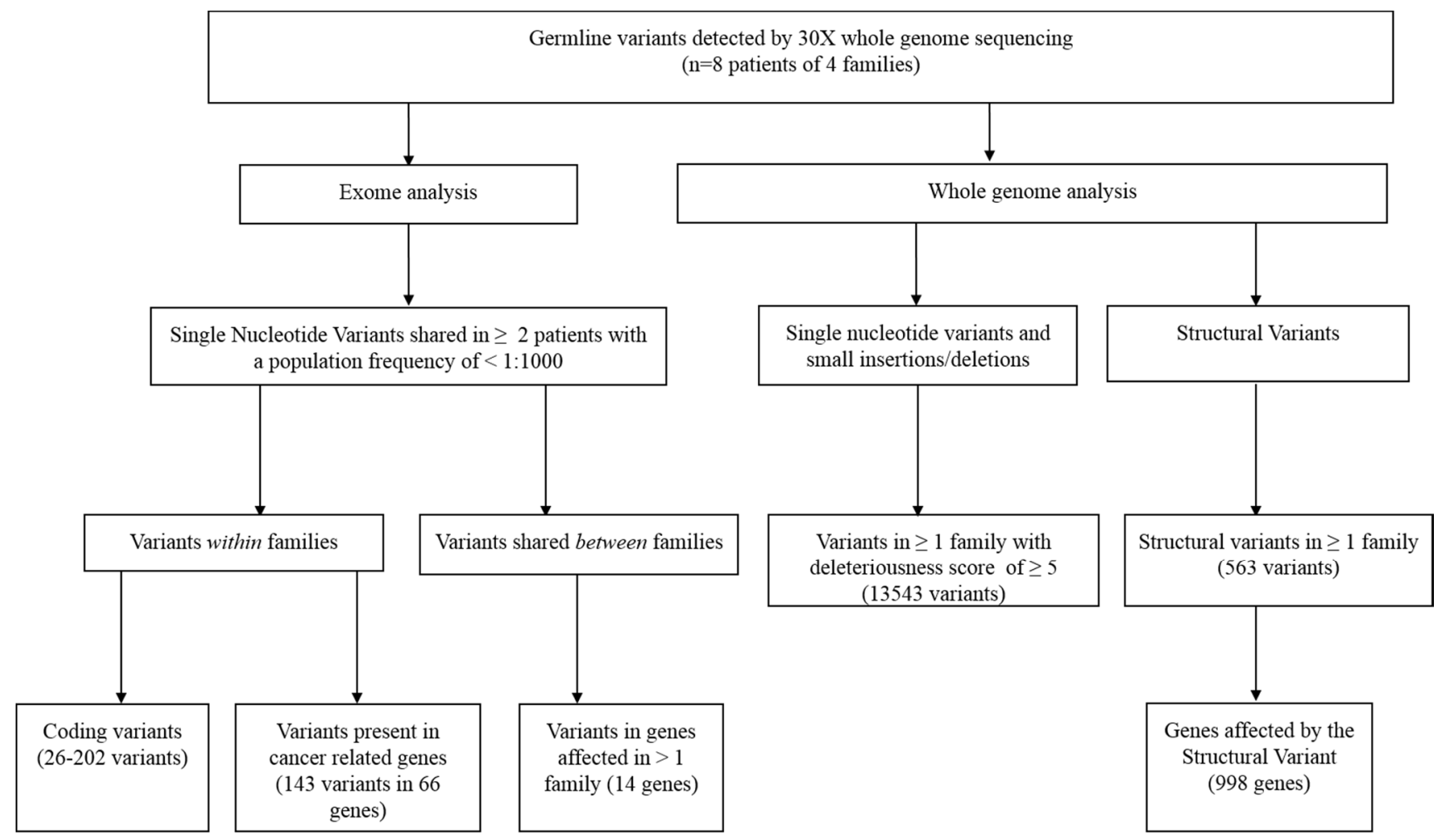

Figure 2. Filter approach.

Subsequently, we investigated genes that were recurrently hit in at least two families, which could point to a molecular mechanism of pathogenesis for AGCT. Across all coding genes, we identified fourteen genes that were affected by identical or different singlenucleotide variants in the same gene among at least two families (Table S1). No genes remained after filtering out variants present in low quality or intronic regions and genes that commonly have false-positive variant calls from next-generation sequencing. 
Table 2. Detected overlapping germline variants per family.

\begin{tabular}{|c|c|c|c|c|}
\hline Family & Gene & Effect & $\begin{array}{l}\text { Variant } \\
\text { (cDNA) }\end{array}$ & $\begin{array}{l}\text { Variant } \\
\text { (Protein) }\end{array}$ \\
\hline \multirow[t]{3}{*}{ A } & HTRA4 & Nonsynonymous & c. $1009 \mathrm{G}>\mathrm{C}$ & p.V337L \\
\hline & $L R P 2$ & Nonsynonymous & c. $2688 \mathrm{C}>\mathrm{G}$ & p.H896Q \\
\hline & PCSK9 & Nonsynonymous & c. $479 \mathrm{G}>\mathrm{A}$ & p.R160Q \\
\hline \multirow[t]{8}{*}{ B } & BMP5 & Nonsynonymous & c. $1291 \mathrm{~T}>\mathrm{C}$ & p.Y431H \\
\hline & CRLF2 & Frameshift & c.496_497delGT & p.N166Yfs*111 \\
\hline & FSCN3 & Nonsynonymous & c. $212 \mathrm{G}>\mathrm{A}$ & p.G71D \\
\hline & HFM1 & Nonsynonymous & c. $4283 \mathrm{~T}>\mathrm{C}$ & p.L1428S \\
\hline & $M E T$ & Nonsynonymous & c. $3409 \mathrm{G}>\mathrm{A}$ & p.G1137R \\
\hline & NOX5 & Nonsynonymous & c. $1600 G>C$ & p.G534R \\
\hline & SPTBN5 & Nonsynonymous & c. $10672 \mathrm{~T}>\mathrm{C}$ & p.W3558R \\
\hline & TEAD2 & Frameshift & c.1286_1287delAT & p.Y429Cfs*55 \\
\hline \multirow[t]{7}{*}{ C } & $C B X 8$ & Nonsynonymous & c. $916 \mathrm{C}>\mathrm{T}$ & p.R306W \\
\hline & HYDIN & Frameshift & c.6584_6585ins59 & p.P2196Ifs*17 \\
\hline & IGSF1 & Nonsynonymous & c. $709 \mathrm{C}>\mathrm{G}$ & p.P237A \\
\hline & LAMA3 & Nonsynonymous & c. $701 \mathrm{~T}>\mathrm{A}$ & p.I234K \\
\hline & PSMD5 & Nonsynonymous & c. $820 \mathrm{G}>\mathrm{A}$ & p.V274M \\
\hline & PXDN & Nonsynonymous & c. $3464 \mathrm{C}>\mathrm{A}$ & p.A1155E \\
\hline & TBP & Frameshift & c.231_234delGCAGinsCAG & p.Q77Hfs*67 \\
\hline \multirow[t]{2}{*}{$\mathrm{D}$} & USP44 & Nonsynonymous & c. $1250 \mathrm{G}>\mathrm{A}$ & p.R417H \\
\hline & RASSF2* & Nonsynonymous & c.389T > A & p.L130Q \\
\hline
\end{tabular}

Reported variants included exonic variants passing quality filters that resulting in a frameshift, stop/start loss, or a nonsynonymous variant predicted to be pathogenic from 3/5 pathogenicity algorithms and candidate genes affected in two families. * Variant did not meet inclusion criteria but was reported as the gene is a tumor suppressor, and the variant is rare and was predicted damaging in $2 / 5$ algorithms.

\subsection{Whole-Genome Analysis}

Genome wide, we found 13,543 single nucleotide variants present in at least 1 family with a CADD PHRED deleteriousness score of $\geq 5$ (Figure 2 and Table S1). Variants with the highest deleteriousness score (CADD PHRED > 30) included frameshift variants predicted to result in loss of function of PIK3C2G (p.Asn1129Thrfs*27), KLHL33, and MYH1 (rs545765873), and three intergenic variants. Furthermore, 52 single nucleotide variants were shared by at least two families. Two single nucleotide variants were present in three families, of which one could not be validated by PCR and was classified as a sequencing artefact (rs1306359244, chr12:103223803, near IGF-1). The other variant (rs781644268, chr12:53833990) was an intergenic variant downstream of AMHR2 and upstream of PRR13 and positioned at a long $\mathrm{T}$ stretch.

A total of 563 structural variants were identified that were shared within at least one family, including 72 structural variants shared by two different families (Figure 2 and Table S1). These structural variants collectively contained 998 individual genes that were affected by these variants. We assessed the genes that were affected in at least two families $(n=104 ;$ Table S1). No gene was affected in more than two families.

AGCTs have characteristic somatic copy number gains and losses that are present in a large proportion of tumors, specifically gain of chromosome 14 and concurrent loss of chromosome 22 [14]. It is unclear whether these structural variants facilitate tumor development, or if also germline copy number variation could contribute to AGCT evolution. We did not identify germline duplications on chromosome 14 or loss on chromosome 22 affecting multiple families.

\subsection{Variants in Genes Associated with Sex Cord-Stromal Tumor or Hereditary Ovarian Cancer}

Genes associated with the development of sex cord-stromal tumors or hereditary ovarian cancer were specifically investigated. No rare $(<1: 1000)$ germline single nucleotide or structural variants were identified within $75 \mathrm{kB}$ of FOXL2, STK11/LKB1, IDH1/2, DICER1, or for $B R C A 2$. A noncoding mutation shared by five individuals across three families 
(rs12938971) was identified $22 \mathrm{~kb}$ downstream of the coding region of $B R C A 1$, however this variant was present in a polyG stretch in a low complexity region and, therefore, likely to be a sequencing artefact. One report showed an AGCT in both a BRCA1 and a BRCA2 series of ovarian cancers. That study investigated individuals with breast and/or ovarian cancer, from families with at least one ovarian cancer diagnosis [27].

\subsection{Comparison with Unrelated AGCT Patients}

We compared the clinical characteristics and germline variants of the patients from the four families to a reference group of 33 unrelated AGCT patients. The mean age of the familial patients at diagnosis was 38 years (range from 17 to 60), significantly lower than the reference group (mean age at diagnosis 51 years, ranging from 29 to 75 , T-test $p$-value $=0.016$ ), and seven of the eight women were premenopausal at the time of diagnosis. Although one of the patients had metastatic disease at diagnosis $(12.5 \% \mathrm{vs} .3 \%$ in the reference group), none of the patients developed a recurrence. The genomic relatedness within the unrelated AGCT patient cohort was predicted to be at least 7 th degree and between the families and the unrelated patients at least 8th degree (Table S2), indicating no relationship between these patients.

Furthermore, we investigated if recurrent or predicted pathogenic single nucleotide variants and structural variants were present in a reference group of unrelated AGCT patients for whom whole-genome sequencing had been performed on germline DNA derived from blood or saliva [14]. Of the 52 single nucleotide variants shared by at least two families in the present study, 26 variants were present in the reference group and eight variants present at least two times in the reference group. No variant was in a coding area of the genome, and manual inspection of the next-generation sequencing reads of the one variant (rs1185417161) seen seven times in our reference AGCT group revealed reads with many mismatches and most likely mapped to the genome incorrectly. Finally, no structural variants present in this study were identified in the reference group. None of the patients in the reference group had a PIK3C2G variant and one patient had a BMP5 variant, although it was not predicted to be pathogenic. A predicted damaging LRP2 p. His896Gln variant was identified in family A. Homozygous pathogenic variants in LRP2 result in Donnai-Barrow syndrome [28], and heterozygous loss-of-function variants are not well tolerated in the general population [29]. Splice variant LRP2 somatic mutations have also been identified previously in AGCTs [30]. We identified thirteen exonic nonsynonymous LRP2 variants in the reference group, of which two were predicted to be pathogenic by $3 / 5$ prediction algorithms (Table S1). One of these variants was identified only once (rs760331558) and the other (rs61995913) was present at an allele frequency of 0.004 . Therefore, no specific mutations were shared, although predicted pathogenic variants in LRP2 were found in both related patients and the reference group.

\section{Discussion}

This study describes the familial occurrence of adult granulosa cell tumors of the ovary. For the first time, the germline DNA of familial AGCT patients was investigated, with the aim to identify an overlapping genetic variant or affected gene that could have contributed to tumor evolvement. The genomic analyses covered both small single nucleotide variants, insertions and deletions, and larger structural variation. We focused on both variation that was shared between families as well as predicted damaging variants present in one family.

Although we did not find a genetic variant that was shared within all four families, we did identify variants in genes that were predicted to be damaging. Of these genes, LRP2 (variant in family A) is involved in lysosomal regulation of lipid metabolism [31]. Additionally, a nonsynonymous coding variant in LRP1 was detected in family C. Although this variant was not predicted to be damaging, LRP1 is also involved in lipid metabolism [31]. Previous studies have shown that the lipid metabolism in granulosa cells plays a vital role during follicular development and is indispensable for oocyte maturation [32-34]. In addition, LRP2 expression is induced by the peroxisome proliferator-activated receptor- 
gamma (PPAR $\gamma)$, a key transcriptional factor regulating lipid metabolism which is widely expressed in granulosa cells [35]. PPAR $\gamma$ activation, combined with inhibition of the Xlinked inhibitor of apoptosis protein (XIAP), has been suggested as a novel therapeutic strategy in AGCTs [36].

Furthermore, we found a predicted damaging PIK3C2G variant in family C (deleteriousness score: 33 , predicted to result in nonsense-mediated decay). PIK3C2G is involved in cell signaling pathways that regulate cell proliferation, survival, and oncogenic transformation, and is altered in $0.58 \%$ of all cancers [37]. An analysis of 4034 cases from The Cancer Genome Atlas identified germline truncating mutations in 34 genes, including PIK3C2G. Germline PIK3C2G truncating variants were associated with cancer predisposition, specifically with ovarian cancer [38].

We also found a very rare predicted damaging heterozygous variant in BMP5 in family B. Bone morphogenetic proteins (BMPs) play an important role in embryonic and postnatal development by regulating cell differentiation, proliferation, and survival, thus maintaining homeostasis during organ and tissue development [39]. BMPs can serve as either oncogenes or tumor suppressors, leading to tumorigenesis and regulating cancer progression [40]. The BMPs are cytokines belonging to the Transforming Growth Factor (TGF)- $\beta$ superfamily, which also includes TGF- $\beta$ s, activin, inhibin, nodal, and myostatin [39]. BMPs activate SMAD pathways, phosphatidylinositol 3-kinase (PI3K)/ AKT, mitogen-activated protein kinase (MAPK), nuclear factor kappa B (NF-kB), and Janus kinase/signal transducer and activator of transcription (JAK/STAT) signaling pathways. A recent study found that TGF- $\beta$ signaling enhances the effect of mutant FOXL2 (c.402C $>$ G) and BMP stimulation (via signaling through $S M A D 1, S M A D 5$ and $S M A D 8$ ) seems to counteract this effect. Moreover, conditional double deletion of $S M A D 1$ and $S M A D 5$ or triple deletion of SMAD1, $S M A D 5, S M A D 8$ led to infertility and granulosa cell tumor development in mice [41]. $B M P 5$ somatic missense mutations are present in $7.7 \%$ of colorectal cases and reduction in BMP5 through loss of function or damaging nonsynonymous variants has been linked to disease progression in cancer [42,43]. Targeting BMPs and their receptors were successful in preventing tumor growth and invasion in preclinical and clinical cancer studies [44].

It is not yet known whether a genetic cause for AGCT exists in these patients, since we could not identify a shared variant or affected locus between the families that could be linked to granulosa cell tumor development. Additionally, the families included many unaffected females, indicating that if an autosomal dominant causal variant is present, it could be a variant with incomplete penetrance. Reduced penetrance may result from a combination of genetic, environmental, and lifestyle factors. This phenomenon can make it challenging for genetic professionals to interpret an individual's family medical history and predict the risk of passing on a genetic condition. However, affected family members from both family A and D are fifth-degree relatives and their environmental and lifestyle factors are therefore likely to differ. Another possibility is a familial detrimental variant in a tumor suppressor gene or a gene involved in granulosa cell proliferation, in which another somatically acquired variant is needed to knock out or reduce function of the gene and initiate tumorigenesis or facilitate tumor development.

However, the identification of four different families, of which three families originate from a country with only 15-20 new cases per year, including first-, second- or fifth-degree relatives with a very rare gynecological tumor makes a genetic germline contribution plausible. The fact that the age of onset in the familial cases was significantly lower than in incidental AGCTs, with the youngest patient being diagnosed at the age of 17 years, strengthens this hypothesis as a disease caused by a germline variant usually manifests at a younger age.

Besides the occurrence of granulosa cell tumors, cases of breast cancer were found in first-degree relatives of the AGCT patients in two families. In these patients, the median age at diagnosis was lower (51.7 years) than the average age at breast cancer diagnosis in the general population (62 years) [45]. A potential link between AGCTs and breast cancer has previously been suggested. A recent study including 1908 AGCT cases found 
a higher observed $(n=79,4.14 \%)$ than expected number of breast cancer cases $(n=27$, $1.41 \%$ ) [46]. Moreover, other studies report a breast cancer rate of 5-10\% among AGCT patients [47-49]. Furthermore, two AGCT patients were subfertile and diagnosed with polycystic ovary syndrome. Several studies reported a possible connection of granulosa cell tumors to subfertility, with improved fertility after surgical removal of the tumor [50]. Granulosa cell tumors at the fertile age were associated with nulliparity and with a clinical presentation of anovulatory infertility, while AGCTs later in life were associated with a normal average fertility pattern. In our study, breast cancer and subfertility were also present in non-affected family members. The co-occurrence of granulosa cell tumors, breast cancer, and/or subfertility in these families may indicate a shared etiology, for example, a genetic predisposition.

It is known that trying to resolve a familial disease can be a daunting task. For example, in familial high grade ovarian carcinoma, the hereditary basis of approximately $50 \%$ of cases is still unexplained. A recent study on suggested familial BRCA1/2 wildtype high grade ovarian cancers found that only $6.6 \%$ of cases could potentially be explained by genes known or suggested to be linked with a higher risk of ovarian cancer [51]. This study reported a high number of individual rare loss of function variants, suggesting that these could be genuine predisposing variants, which is in agreement with our findings (rare loss of function variants in PIK3C2G, KLHL33, and MYH1).

The ongoing advances in the field of whole-genome sequencing data analysis may lead to novel insights and may resolve unexplained familial cancer cases. Nonetheless, the identification of a causal germline variant may not have direct clinical implications as, due to the rarity of the disease, genetic testing for AGCT patients or their family members in general may not be necessary. Moreover, the identification of four families and one previously reported family indicates that the vast majority of cases are sporadic rather than familial. However, identification of a predisposing genetic factor could help to unravel the pathological mechanism of AGCTs.

\section{Conclusions}

Adult granulosa cell tumors can occur in familial clusters and could potentially be hereditary. We did not identify an overlapping genetic variant or affected genetic locus that may explain a genetic predisposition for AGCT in the four investigated families. In these families, the age of AGCT diagnosis was lower than in unrelated cases, and breast cancer, PCOS, and subfertility were reported in these families, suggesting potential shared etiologic factors.

Supplementary Materials: The following are available online at https:/ /www.mdpi.com/article/10 .3390 / cancers13102430/s1, Table S1: Variant Analysis, Table S2: Genomic relatedness of families.

Author Contributions: J.F.R.: Conceptualization, Methodology, Formal analysis, Investigation, Writing-Original Draft, Visualization, Project Administration. J.K.: Methodology, Software, Formal analysis, Investigation, Data Curation, Writing-Review and Editing. W.K.: Formal analysis, Investigation, Writing-Review \& Editing. M.G.E.M.A.: Conceptualization, Writing-Review and Editing, Visualization. K.E.: Conceptualization, Investigation, Resources, Writing-Review and Editing. J.M.J.P.: Resources, Writing-Review and Editing. C.D.d.K.: Resources, Writing-Review and Editing. R.H.M.V.: Conceptualization, Funding acquisition, Writing-Review and Editing. G.v.H.: Methodology, Formal analysis, Writing-Original Draft, Supervision. R.P.Z.: Conceptualization, Methodology, Writing-Original Draft, Supervision, Funding acquisition. G.R.M.: Conceptualization, Methodology, Software, Formal analysis, Investigation, Data Curation, Writing-Original Draft, Supervision. All authors have read and agreed to the published version of the manuscript.

Funding: This research was carried out with financial support of the Granulosa Fund Philine van Esch. This work was further supported in part by a grant from the Dutch Cancer Society: JK was funded by KWF-RUG 2017-11352. 
Institutional Review Board Statement: The study was conducted according to the guidelines of the Declaration of Helsinki, and ethical approval was obtained by the Institutional Review Board of the University Medical Center Utrecht (UMCU METC 17-868).

Informed Consent Statement: Informed consent was obtained from all subjects involved in the study.

Data Availability Statement: WGS Binary Alignment Map (BAM) files are available through controlled access at the European Genome-phenome Archive (EGA), hosted at the EBI and the CRG (https:/ /ega-archive.org, 8 January 2021), with EGA dataset ID: EGAD00001006780. Requests for data access will be evaluated by the UMCU Department of Genetics Data Access Committee (EGAC00001000432) and transferred on completion of a material transfer agreement and authorization by the medical ethical committee of the UMCU to ensure compliance with the Dutch 'medical research involving human subjects' act.

Acknowledgments: The authors wish to thank the Granulosa Fund Philine van Esch, which provided funding that was critical to this project. The authors gratefully thank Eric Hennekam, Noah Scheffer, and Martin Elferink for evaluating relatedness using genealogy and genomic analysis. The authors further gratefully thank Ralph Hermans, Kirsten Smeets, Tjalling Bosse, Carrie Langstraat, and Julian Schink for providing clinical data. This work was further supported in part by a grant from the Dutch. Cancer Society: JK was funded by KWF-RUG 2017-11352.

Conflicts of Interest: The authors declare no conflict of interest.

\section{References}

1. Siegel, R.L.; Miller, K.D.; Jemal, A. Cancer statistics, 2020. CA Cancer J. Clin. 2020, 70, 7-30. [CrossRef] [PubMed]

2. Schumer, S.T.; Cannistra, S.A. Granulosa cell tumor of the ovary. J. Clin. Oncol. 2003, 21, 1180-1189. [CrossRef] [PubMed]

3. Gainder, S.; Kaur, J.; Siwatch, S.; Gupta, N. Adult Granulosa Cell Tumor: A Sinister Differential for Clomiphene-resistant Infertility. J. Hum. Reprod. Sci. 2018, 11, 190-192.

4. Lappohn, R.E.; Burger, H.G.; Bouma, J.; Bangah, M.; Krans, M.; de Bruijn, H.W. Inhibin as a marker for granulosa-cell tumors. N. Engl. J. Med. 1989, 321, 790-793. [CrossRef] [PubMed]

5. Karnezis, A.N.; Cho, K.R.; Gilks, C.B.; Pearce, C.L.; Huntsman, D.G. The disparate origins of ovarian cancers: Pathogenesis and prevention strategies. Nat. Rev. Cancer 2016, 17, 65-74. [CrossRef] [PubMed]

6. Zhang, S.; Royer, R.; Li, S.; McLaughlin, J.R.; Rosen, B.; Risch, H.A.; Fan, I.; Bradley, L.; Shaw, P.A.; Narod, S.A. Frequencies of BRCA1 and BRCA2 mutations among 1,342 unselected patients with invasive ovarian cancer. Gynecol. Oncol. 2011, 121, 353-357. [CrossRef] [PubMed]

7. Giardiello, F.M.; Brensinger, J.D.; Tersmette, A.C.; Goodman, S.N.; Petersen, G.M.; Booker, S.V.; Cruz-Correa, M.; Offerhaus, J.A. Very high risk of cancer in familial Peutz-Jeghers syndrome. Gastroenterology 2000, 119, 1447-1453. [CrossRef]

8. Online Mendelian Inheritance in Man. Available online: https:/ /www.omim.org/entry / 601200 (accessed on 2 February 2021).

9. Fuller, P.J.; Leung, D.; Chu, S. Genetics and genomics of ovarian sex cord-stromal tumors. Clin. Genet. 2017, 91, 285-291. [CrossRef]

10. Shah, S.P.; Kobel, M.; Senz, J.; Morin, R.D.; Clarke, B.A.; Wiegand, K.C.; Leung, G.; Zayed, A.; Mehl, E.; Kalloger, S.E.; et al. Mutation of FOXL2 in granulosa-cell tumors of the ovary. N. Engl. J. Med. 2009, 360, 2719-2729. [CrossRef] [PubMed]

11. Kim, J.-H.; Yoon, S.; Park, M.; Park, H.-O.; Ko, J.-J.; Lee, K.; Bae, J. Differential apoptotic activities of wild-type FOXL2 and the adult-type granulosa cell tumor-associated mutant FOXL2 (C134W). Oncogene 2011, 30, 1653-1663. [CrossRef]

12. Online Mendelian Inheritance in Man. Available online: https://www.omim.org/entry/110100 (accessed on 2 February 2021).

13. Stevens, T.A.; Brown, J.; Zander, D.S.; Bevers, M.W.; Gershenson, D.M.; Ramondetta, L.M. Adult granulosa cell tumors of the ovary in two first-degree relatives. Gynecol. Oncol. 2005, 98, 502-505. [CrossRef]

14. Roze, J.; Monroe, G.; Kutzera, J.; Groeneweg, J.; Stelloo, E.; Paijens, S.; Nijman, H.; van Meurs, H.; van Lonkhuijzen, L.; Piek, J.; et al. Whole genome analysis of ovarian granulosa cell tumors reveals tumor heterogeneity and a high- grade tp53-specific subgroup. Cancers 2020, 12, 1308. [CrossRef] [PubMed]

15. Roze, J.; Groeneweg, J.; Monroe, G.; Fransen, I.; Gultekin, M.; Zweemer, R.; Verheijen, R.H.M. Patient engagement in research on rare gynecological tumors. Int. J. Gynecol. Cancer 2020, ijgc-2020. [CrossRef] [PubMed]

16. Twine, N.; Szul, P.; Henden, L.; McCann, E.; Blair, I.; Williams, K.; Bauer, D. TRIBES: A user-friendly pipeline for relatedness detection and disease gene discovery. bioRxiv 2019, 1-3. [CrossRef]

17. Github. Available online: https://github.com/UMCUGenetics/IAP (accessed on 11 March 2021).

18. Rausch, T.; Zichner, T.; Schlattl, A.; Stutz, A.M.; Benes, V.; Korbel, J.O. DELLY: Structural variant discovery by integrated paired-end and split-read analysis. Bioinformatics 2012, 28, i333-i339. [CrossRef] [PubMed]

19. Lek, M.; Karczewski, K.J.; Minikel, E.V.; Samocha, K.E.; Banks, E.; Fennell, T.; O’Donnell-Luria, A.H.; Ware, J.S.; Hill, A.J.; Cummings, B.B.; et al. Analysis of protein-coding genetic variation in 60,706 humans. Nature 2016, 536, 285-291. [CrossRef] 
20. Karczewski, K.J.; Francioli, L.C.; Tiao, G.; Cummings, B.B.; Alföldi, J.; Wang, Q.; Collins, R.L.; Laricchia, K.M.; Ganna, A.; Birnbaum, D.P.; et al. The mutational constraint spectrum quantified from variation in 141,456 humans. Nature 2020,581, 434-443. [CrossRef]

21. The 1000 Genomes Project Consortium. A global reference for human genetic variation. Nature 2015, 526, 68-74. [CrossRef]

22. Boomsma, D.I.; Wijmenga, C.; Slagboom, E.P.; Swertz, M.A.; Karssen, L.C.; Abdellaoui, A.; Ye, K.; Guryev, V.; Vermaat, M.; van Dijk, F.; et al. The Genome of the Netherlands: Design, and project goals. Eur. J. Hum. Genet. 2014, 22, 221-227. [CrossRef]

23. Github. Available online: https://github.com/hartwigmedical/hmftools (accessed on 11 March 2021).

24. Kircher, M.; Witten, D.M.; Jain, P.; O’Roak, B.J.; Cooper, G.M.; Shendure, J. A general framework for estimating the relative pathogenicity of human genetic variants. Nat. Genet. 2014, 46, 310-315. [CrossRef]

25. Collins, R.L.; Brand, H.; Karczewski, K.J.; Zhao, X.; Alföldi, J.; Francioli, L.C.; Khera, A.V.; Lowther, C.; Gauthier, L.D.; Wang, H.; et al. A structural variation reference for medical and population genetics. Nature 2020, 581, 444-451. [CrossRef] [PubMed]

26. Bioconductor. Available online: https://www.bioconductor.org/packages/release/bioc/html/StructuralVariantAnnotation.html (accessed on 4 February 2021).

27. Evans, D.; Young, K.; Bulman, M.; Shenton, A.; Wallace, A.; Lalloo, F. Probability of BRCA1/2 mutation varies with ovarian histology: Results from screening 442 ovarian cancer families. Clin. Genet. 2008, 73, 338-345. [CrossRef]

28. Online Mendelian Inheritance in Man. Available online: https://www.omim.org/entry/222448 (accessed on 2 February 2021).

29. The Genome Aggregation Database. Available online: https://gnomad.broadinstitute.org/ (accessed on 5 February 2021).

30. Alexiadis, M.; Rowley, S.M.; Chu, S.; Leung, D.T.H.; Stewart, C.J.R.; Amarasinghe, K.C.; Campbell, I.G.; Fuller, P.J. Mutational landscape of ovarian adult granulosa cell tumors from whole exome and targeted TERT promoter sequencing. Mol. Cancer Res. 2019, 17, 177-185. [CrossRef]

31. Staudt, C.; Puissant, E.; Boonen, M. Subcellular Trafficking of Mammalian Lysosomal Proteins: An Extended View. Int. J. Mol. Sci. 2016, 18, 47. [CrossRef] [PubMed]

32. Hu, W.; Qiao, J. Expression and regulation of adipocyte fatty acid binding protein in granulosa cells and its relation with clinical characteristics of polycystic ovary syndrome. Endocrine 2011, 40, 196-202. [CrossRef]

33. Elis, S.; Desmarchais, A.; Maillard, V.; Uzbekova, S.; Monget, P.; Dupont, J. Cell proliferation and progesterone synthesis depend on lipid metabolism in bovine granulosa cells. Theriogenology 2015, 83, 840-853. [CrossRef] [PubMed]

34. Gao, S.; Gan, X.; He, H.; Hu, S.; Deng, Y.; Chen, X.; Li, L.; Hu, J.; Li, L.; Wang, J. Dynamic characteristics of lipid metabolism in cultured granulosa cells from geese follicles at different developmental stages. Biosci. Rep. 2019, 39. [CrossRef] [PubMed]

35. Cabezas, F.; Lagos, J.; Céspedes, C.; Vio, C.P.; Bronfman, M.; Marzolo, M.-P. Megalin/LRP2 Expression Is Induced by Peroxisome Proliferator-Activated Receptor -Alpha and -Gamma: Implications for PPARs' Roles in Renal Function. PLoS ONE 2011, 6, e16794. [CrossRef]

36. Leung, D.T.H.; Nguyen, T.; Oliver, E.M.; Matti, J.; Alexiadis, M.; Silke, J.; Jobling, T.W.; Fuller, P.J.; Chu, S. Combined PPAR $\gamma$ Activation and XIAP Inhibition as a Potential Therapeutic Strategy for Ovarian Granulosa Cell Tumors. Mol. Cancer Ther. 2019, 18, 364-375. [CrossRef]

37. AACR Project GENIE: Powering Precision Medicine through an International Consortium. Cancer Discov. $2017,7,818-831$. [CrossRef]

38. Lu, C.; Xie, M.; Wendl, M.C.; Wang, J.; McLellan, M.D.; Leiserson, M.D.M.; Huang, K.; Wyczalkowski, M.A.; Jayasinghe, R.; Banerjee, T.; et al. Patterns and functional implications of rare germline variants across 12 cancer types. Nat. Commun. 2015, 6, 10086. [CrossRef] [PubMed]

39. Miyazono, K.; Kamiya, Y.; Morikawa, M. Bone morphogenetic protein receptors and signal transduction. J. Biochem. 2010, 147, 35-51. [CrossRef] [PubMed]

40. Blanco Calvo, M.; Bolós Fernández, V.; Medina Villaamil, V.; Aparicio Gallego, G.; Díaz Prado, S.; Grande Pulido, E. Biology of BMP signalling and cancer. Clin. Transl. Oncol. 2009, 11, 126-137. [CrossRef] [PubMed]

41. Pangas, S.A.; Li, X.; Umans, L.; Zwijsen, A.; Huylebroeck, D.; Gutierrez, C.; Wang, D.; Martin, J.F.; Jamin, S.P.; Behringer, R.R.; et al. Conditional Deletion of Smad1 and Smad5 in Somatic Cells of Male and Female Gonads Leads to Metastatic Tumor Development in Mice. Mol. Cell. Biol. 2008, 28, 248-257. [CrossRef]

42. Karim, M.A.; Samad, A.; Adhikari, U.K.; Kader, M.A.; Kabir, M.M.; Islam, M.A.; Hasan, M.N. A Multi-Omics Analysis of Bone Morphogenetic Protein 5 (BMP5) mRNA Expression and Clinical Prognostic Outcomes in Different Cancers Using Bioinformatics Approaches. Biomedicines 2020, 8, 19. [CrossRef]

43. Chen, E.; Yang, F.; He, H.; Li, Q.; Zhang, W.; Xing, J.; Zhu, Z.; Jiang, J.; Wang, H.; Zhao, X.; et al. Alteration of tumor suppressor BMP5 in sporadic colorectal cancer: A genomic and transcriptomic profiling based study. Mol. Cancer 2018, 17, 176. [CrossRef]

44. Zhang, L.; Ye, Y.; Long, X.; Xiao, P.; Ren, X.; Yu, J. BMP signaling and its paradoxical effects in tumorigenesis and dissemination. Oncotarget 2016, 7, 78206-78218. [CrossRef]

45. SEER Cancer Statistics Review (CSR) 1975-2017. Available online: https://seer.cancer.gov/csr/1975_2017/ (accessed on 2 February 2021).

46. Nasioudis, D.; Wilson, E.; Mastroyannis, S.A.; Sisti, G.; Haggerty, A.F.; Ko, E.M.; Latif, N.A. Increased Risk of Breast and Uterine Cancer Among Women With Ovarian Granulosa Cell Tumors. Anticancer Res. 2019, 39, 4971-4975. [CrossRef]

47. Hammer, A.; Lauszus, F.F.; Petersen, A.C. Ovarian granulosa cell tumor and increased risk of breast cancer. Acta Obstet. Gynecol. Scand. 2013, 92, 1422-1425. [CrossRef] 
48. Bryk, S.; Pukkala, E.; Färkkilä, A.; Heikinheimo, M.; Unkila-Kallio, L.; Riska, A. Other Primary Malignancies Among Women With Adult-Type Ovarian Granulosa Cell Tumors. Int. J. Gynecol. Cancer 2018, 28, 1529-1534. [CrossRef]

49. Meisel, J.L.; Hyman, D.M.; Jotwani, A.; Zhou, Q.; Abu-Rustum, N.R.; Iasonos, A.; Pike, M.C.; Aghajanian, C. The role of systemic chemotherapy in the management of granulosa cell tumors. Gynecol. Oncol. 2015, 136, 505-511. [CrossRef] [PubMed]

50. Unkila-Kallio, L.; Tiitinen, A.; Wahlström, T.; Lehtovirta, P.; Leminen, A. Reproductive features in women developing ovarian granulosa cell tumour at a fertile age. Hum. Reprod. 2000, 15, 589-593. [CrossRef] [PubMed]

51. Subramanian, D.N.; Zethoven, M.; McInerny, S.; Morgan, J.A.; Rowley, S.M.; Lee, J.E.A.; Li, N.; Gorringe, K.L.; James, P.A.; Campbell, I.G. Exome sequencing of familial high-grade serous ovarian carcinoma reveals heterogeneity for rare candidate susceptibility genes. Nat. Commun. 2020, 11, 1640. [CrossRef] [PubMed] 